\title{
BODY COMPOSITION IN OLDER PERSONS RESIDING IN RURAL AND URBAN AREAS IN NORTHEASTERN POLAND: A COMPARATIVE ANALYSIS
}

\section{SKŁAD CIAŁA SENIORÓW ŻYJĄCYCH NA WSI I W MIEŚCIE W REGIONIE POLSKI PÓŁNOCNO-WSCHODNIEJ: ANALIZA PORÓWNAWCZA}

\author{
Aneta Anna Omelan ${ }^{1(A, B, D, E, F)}$, Ernest Bielinis $^{2(C, D)}$, \\ Andrzej Rutkowski ${ }^{3(B, D)}$, Robert Podstawski ${ }^{1(\mathrm{E}, \mathrm{F})}$ \\ ${ }^{1}$ Department of Tourism, Recreation and Ecology, University of Warmia and Mazury in Olsztyn, Poland \\ ${ }^{2}$ Department of Forestry and Forest Ecology, University of Warmia and Mazury in Olsztyn, Poland \\ ${ }^{3}$ Physical Activity and Security Education Group, Complex of Construction Schools, Olsztyn, Poland
}

Authors' contribution Wkład autorów:

A. Study design/planning zaplanowanie badań B. Data collection/entry zebranie danych C. Data analysis/statistics dane - analiza i statystyki D. Data interpretation interpretacja danych E. Preparation of manuscript przygotowanie artykułu F. Literature analysis/search wyszukiwanie i analiza literatury G. Funds collection zebranie funduszy
Tables: 3

Figures: 0

References: 28

Submitted: 2020 May 14

Accepted: 2020 Jul 24

\section{Summary}

Background. Older people differ in their physical, mental and social functioning. As we age, changes in the body are reflected in body composition. These changes allow for the assessment of the risk of many diseases. The aim of this study was to analyze the body composition of older rural and urban residents in view of health problems associated with aging. Material and methods. The study involved a total of 627 people aged 60 to 90 years. A questionnaire study and body composition analysis performed with the InBody 270 analyzer were used to assess the parameters in the study. Differences were assessed with two-tailed chi-square tests and the Mann-Whitney U test. Results. A significant $(\mathrm{p}<0.001)$ difference was noted between the body weight of urban $(73.39 \mathrm{~kg})$ and rural dwellers $(79.06 \mathrm{~kg})$. Older women residing in cities were significantly $(\mathrm{p}<0.001)$ less heavy than female rural dwellers. Rural residents were characterized by significantly higher $(p=0.001)$ values of Body Fat Mass (BFM), Fat-Free Mass $(F F M)(p<0.001)$, Skeletal Muscle Mass (SMM) $(p<0.001)$ and Body Mass Index (BMI) $(p<0.001)$. Conclusions. Older rural residents have less optimal body composition parameters than their urban peers. Women residing in rural areas are characterized by the highest BMI, PBF, VFL and degree of obesity, and they can be regarded as the highest risk group of age-related diseases and lower quality of life. Body composition parameters are most favorable in aging males residing in urban areas, who belong to the lowest risk group for disease. The participants' BMI was correlated with the place of residence, membership in senior organizations and marital status.

Keywords: obesity, health, body composition, aging

\section{Streszczenie}

Wprowadzenie. Osoby starsze różnią się między sobą pod względem funkcjonowania fizycznego, psychicznego i społecznego. Zmiany zachodzące $\mathrm{w}$ ich organizmach $\mathrm{w}$ procesie starzenia odzwierciedlają się w składzie ciała. Pozwala to na ocenę ryzyka zachorowania na wiele chorób. Celem badań była ocena i porównanie składu ciała osób starszych mieszkających na wsi i w mieście pod kątem zagrożeń zdrowotnych wynikających z zaawansowanego wieku. Materiał i metody. W badaniu wzięło udział 627 osób w wieku 60-90 lat. Zastosowano badanie sondażowe oraz analizę składu ciała przy użyciu analizatora składu ciała InBody 270 . Różnice pomiędzy mieszkańcami wsi i miast oraz kobietami i mężczyznami oceniono za pomocą testu chi-kwadrat oraz testu U-Mann-Whiteney. Wyniki. Zanotowano statystycznie istotną różnicę $(\mathrm{p}<0,001)$ pomiedzy masa ciała seniorów mieszkających $w$ mieście $(73,39 \mathrm{~kg})$ i na wsi $(79,06$ $\mathrm{kg})$. Starsze kobiety mieszkające w mieście miały statystycznie istotnie $(\mathrm{p}<0,001)$ mniejszą masę ciała niż kobiety mieszkające na wsi. Mieszkańcy wsi charakteryzowali się znaczaco wyższymi $(\mathrm{p}=0,001)$ wartościami tłuszczowej masy ciała (BFM), beztłuszczowej masy ciała (FFM) $(p<0,001)$, masy mięśni szkieletowych $(S M M)(p<0,001)$ oraz wskaźnikiem masy ciała (BMI) (p<0,001). Wnioski. Mieszkańcy wsi maja gorsze parametry składników ciała niż ich miejscy rówieśnicy. Grupą największego ryzyka zdrowotnego oraz najniższej jakości życia są wiejskie kobiety, które mają najwyższe BMI, PBF, VFL oraz najwyższy stopień otyłości. Grupą najmniejszego ryzyka zdrowotnego sa starsi mężczyźni z miast, którzy charakteryzują się najkorzystniejszymi parametrami składu ciała. BMI badanych seniorów wykazuje związek z miejscem zamieszkania, przynależnością do organizacji seniorskiej oraz stanem cywilnym.

Słowa kluczowe: otyłość, zdrowie, skład ciała, starość

Omelan AA, Bielinis E, Rutkowski A, Podstawski R. Body composition in older persons residing in rural and urban areas in northeastern Poland: a comparative analysis. Health Prob Civil. 2020; 14(4): 266-274. https://doi.org/10.5114/hpc.2020.97680

Address for correspondence / Adres korespondencyjny: Aneta Anna Omelan, Department of Tourism, Recreation and Ecology, University of Warmia and Mazury in Olsztyn, Michała Oczapowskiego 5, 10-719 Olsztyn, Poland, e-mail: aneta.omelan@uwm.edu.pl, phone: +48 895233456

ORCID: Aneta Anna Omelan https://orcid.org/0000-0002-3495-8837, Ernest Bielinis https://orcid.org/0000-0002-6384-4898,

Robert Podstawski https://orcid.org/0000-0002-1492-252X

Copyright: (C) Pope John Paul II State School of Higher Education in Biała Podlaska, Aneta Anna Omelan, Ernest Bielinis, Andrzej Rutkowski, Robert Podstawski. This is an Open Access journal, all articles are distributed under the terms of the Creative Commons Attribution-NonCommercial-ShareAlike 4.0 International (CC BY-NC-SA 4.0) License (http://creativecommons.org/licenses/by-nc-sa/4.0/), allowing third parties to copy and redistribute the material in any medium or format and to remix, transform, and build upon the material, provided the original work is properly cited and states its license. 


\section{Introduction}

Recent decades have witnessed a considerable increase in life expectancy in many countries around the world. This also applies to Poland where the size of the $>60$ (or $60+$ ) population is estimated to be 8.5 million (22\% of Poland's total population) [1]. Aging is an irreversible process, and the number of older persons will continue to increase, which will influence the functioning of entire societies in various areas, from residential construction to services and Internet access [2]. Aging is accompanied by a progressive deterioration of physical, psychological and social functions. It is an irreversible process, but it does not proceed identically in all people. Older people differ in levels of physical, psychological and social activity, and the physiological changes associated with age are reflected in their body composition. Body composition analyses support assessments of risk factors for disease, and their results can be used in health protection and in programmes aiming to replace unhealthy habits with behaviors that promote health and overall well-being.

Northeastern Poland has the highest population of older people in the country. Considerable differences are noted between older people living in urban versus rural areas. Rural residents aged $>60$ account for $36.1 \%$ of the Polish elderly population [3]. This group remains poorly investigated, particularly with regards to health. In rural areas, a combination of unique health problems, resource shortages, demographic characteristics, cultural behaviors and economic concerns influence the health of local residents [4]. These factors can influence body composition. In this study, the body composition of older rural residents was analyzed and compared to that of elderly urban dwellers.

\section{Material and methods}

The study involved 627 older residents (aged >60) in rural and urban areas in northeastern Poland. The analyzed population was selected by purposive-probability sampling. Rural residents were selected in areas with a predominantly agricultural function, situated remotely from large cities. The interviewers were able to survey all respondents in person (face-to-face). All procedures performed in this study were in accordance with the ethical standards of the Polish Committee of Ethics in Science and with the 1964 Helsinki declaration and its later amendments. The research was been approved by the Scientific Research Ethics Committee of the University of Warmia and Mazury in Olsztyn (Decision No. 6/2018).

The respondents' body composition was determined by bioelectrical impedance analysis (BIA) using the InBody 270 analyzer. BIA is a fast (several minutes), non-invasive, cheap and reproducible method of testing body composition. It can be used in both healthy and ill people of any age category. It consists of measuring the total resultant electrical resistance of the body, which is a derivative of resistance (passive resistance) and reactance (active resistance) using a set of surface electrodes connected to a computer analyzer and using current of a given frequency and intensity $[5,6]$.

Data were analyzed in the SPSS v. 23.0 programme (SPSS Inc., Chicago, IL). The participants were surveyed using a questionnaire to obtain information about their socioeconomic status (the Pen and Paper Personal InterviewPAPI) (Table 1). Physical activity (PA) levels were measured with the International Physical Activity Questionnaire (IPAQ) adapted to older respondents. Urban-rural and male-female differences were assessed with two-tailed chi-square tests and the Mann-Whitney U test. The correlations between the main observed and control variables were determined in a reliability analysis, and the results were used to test the research hypotheses in ordinary least squares (OLS) regression analysis. The relationships between BMI values vs. urbanization indicators and socioeconomic factors (adjusted for age and sex) were tested with the use of regression models developed in SPSS. Separate models were built for men and women. The developed models illustrate the variance in BMI when each independent variable was added. Non-significant variables were automatically rejected. The level of statistical significance was $p<0.05$ for the chi-square test, t-test, and Mann-Whitney $\mathrm{U}$ test, and $p<0.05$ for regression analyses. 


\begin{tabular}{|c|c|c|c|c|c|c|c|c|c|c|c|c|c|c|c|c|c|c|c|c|c|c|c|}
\hline \multirow{3}{*}{ 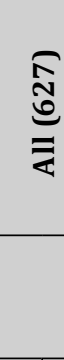 } & की & $\begin{array}{l}0 \\
⿱ \\
1 \\
0\end{array}$ & $\begin{array}{l}\stackrel{2}{n} \\
\tilde{m} \\
\infty\end{array}$ & 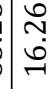 & I & & $\begin{array}{l}m \\
\stackrel{m}{\sim} \\
\end{array}$ & $\begin{array}{l}\vec{n} \\
\text { o. } \\
\dot{+}\end{array}$ & $\begin{array}{l}\stackrel{\sigma}{\vec{z}} \\
\overrightarrow{+}\end{array}$ & $\begin{array}{l}\hat{m} \\
\dot{N}\end{array}$ & & \begin{tabular}{l}
$\infty$ \\
\multirow{+}{*}{} \\
\multirow{+}{*}{}
\end{tabular} & \begin{tabular}{l|l}
\multirow{2}{0}{} \\
$\dot{0}$ \\
$\dot{m}$
\end{tabular} & 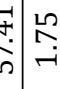 & & 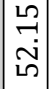 & 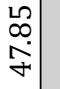 & & $\begin{array}{l}\infty \\
n \\
n \\
m \\
m\end{array}$ & & & \begin{tabular}{l|l}
8 & 7 \\
0 \\
$\infty$ \\
+ \\
+
\end{tabular} & \\
\hline & $\begin{array}{l}\text { ॠ } \\
\sum \\
\Sigma\end{array}$ & 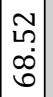 & กิ & $\underset{\sigma}{\stackrel{N}{\sigma}}$ & $m$ & & 号 & 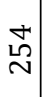 & $\infty$ & $\stackrel{\vec{m}}{\vec{m}}$ & & $\vec{m}$ & $\underset{N}{N} \mid$ & $\begin{array}{l}b \\
b \\
n\end{array}$ & & 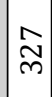 & 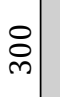 & ָิ & $\begin{array}{l}\infty \\
\stackrel{\varpi}{二}\end{array}$ & & & 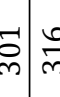 & \\
\hline & $\underbrace{\infty}_{2} \approx$ & \begin{tabular}{l} 
L \\
$\stackrel{2}{2}$ \\
\multirow{2}{0}{}
\end{tabular} & $\bar{c}$ & & & & $\begin{array}{l}\overrightarrow{8} \\
\dot{0} \\
\dot{v}\end{array}$ & & & & & b. & & & & $\begin{array}{l}\text { oे } \\
m \\
0\end{array}$ & & $\begin{array}{l}\overrightarrow{8} \\
0 \\
\dot{0} \\
\mathrm{v}\end{array}$ & & & & & \\
\hline \multirow{4}{*}{ 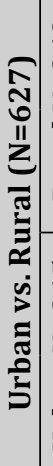 } & के & 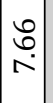 & $\hat{m}$ & $\mid \begin{array}{l}\infty \\
\infty \\
\infty\end{array}$ & fั & & 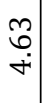 & $\begin{array}{l}0 \\
+ \\
\vdots \\
\sigma\end{array}$ & $\begin{array}{l}\stackrel{?}{0} \\
\hat{\sigma}\end{array}$ & $\begin{array}{l}\stackrel{2}{0} \\
\dot{a} \\
\vec{N}\end{array}$ & & 7 & 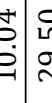 & 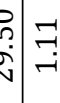 & & $\begin{array}{l}\infty \\
\infty \\
\text { N }\end{array}$ & 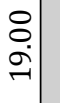 & $\begin{array}{l}0 \\
\infty \\
\stackrel{N}{N}\end{array}$ & $\mid \begin{array}{l}2 \\
2 \\
\infty \\
\sigma\end{array}$ & & & 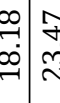 & \\
\hline & $\begin{array}{l}\bar{\varpi} \\
\sum_{\Sigma}^{\infty}\end{array}$ & 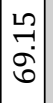 & 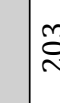 & L & $m$ & & $\stackrel{\sim}{\mathrm{N}}$ & ริ & $\mathcal{F}$ & $\stackrel{\widetilde{N}}{\rightarrow}$ & r & $-\hat{v}$ & \begin{tabular}{lll}
0 & \multicolumn{2}{c}{} \\
\end{tabular} & $\begin{array}{r}\underbrace{0} \\
-1\end{array}$ & & $\stackrel{m}{\sim}$ & $\vec{\xi}$ & $\underset{\sim}{\Psi}$ & $\vec{\exists}$ & & $\rightarrow$ & $\underset{7}{\exists}$ & \\
\hline & คิ & $\begin{array}{l}0 \\
\text { †े } \\
\text { in }\end{array}$ & $\begin{array}{l}\infty \\
\infty \\
0\end{array}$ & $\stackrel{m}{m}$ & ' & & $\begin{array}{l}0 \\
m \\
\vec{a}\end{array}$ & $\begin{array}{l}\stackrel{ }{-} \\
\vec{m} \\
\vec{m}\end{array}$ & 号 & $\begin{array}{c}\tilde{N} \\
\tilde{O}\end{array}$ & & 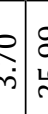 & 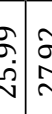 & র̦ & & $\begin{array}{l}\vec{m} \\
\stackrel{\sim}{\sim} \\
\stackrel{N}{2}\end{array}$ & $\begin{array}{l}0 \\
\infty \\
\infty \\
\infty \\
\sim \\
\sim\end{array}$ & $\begin{array}{l}\vec{b} \\
\dot{\rho} \\
\dot{\sigma}\end{array}$ & $\begin{array}{l}0 \\
0 \\
i \\
\end{array}$ & & & 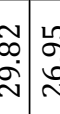 & \\
\hline & 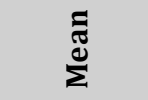 & $\begin{array}{l}0 \\
\vdots \\
0 \\
0 \\
0\end{array}$ & $\frac{\vec{m}}{m}$ & I & ' & & $\overrightarrow{\mathbb{I}}$ & $\stackrel{2}{\sigma} \underset{\sigma}{\sigma}$ & f & $\sim$ & & N) & 仓ી & $=8$ & & 离 & $\begin{array}{l}\vec{\infty} \\
\stackrel{-1}{a}\end{array}$ & 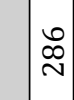 & 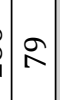 & & $\sigma$ & 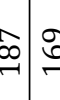 & \\
\hline \multirow{5}{*}{ 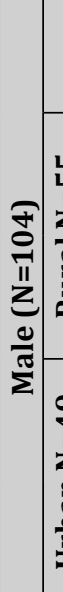 } & 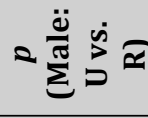 & $\begin{array}{c}\vec{F} \\
\bar{m} \\
0 \\
0\end{array}$ & 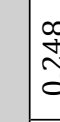 & & & & $\begin{array}{l}8 \\
\vdots \\
\vdots \\
0\end{array}$ & & & & & : & & & & 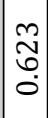 & & : & & & 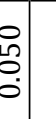 & & \\
\hline & की & 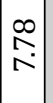 & $\underset{z}{q}$ & $\begin{array}{l}\dot{\sigma} \\
\sigma \\
\sigma\end{array}$ & ' & & $\begin{array}{l}\stackrel{0}{ } \\
\stackrel{1}{\circ}\end{array}$ & 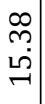 & $\begin{array}{l}\stackrel{o}{0} \\
\stackrel{0}{0}\end{array}$ & $\begin{array}{l}\stackrel{\bullet}{\circ} \\
\stackrel{+}{+}\end{array}$ & $\mathfrak{z}$ & 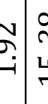 & 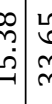 & 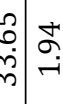 & & 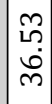 & 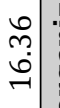 & 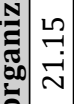 & $\begin{array}{c}n \\
\\
m\end{array}$ & & & 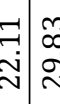 & \\
\hline & 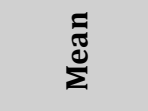 & 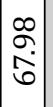 & & 웅 & & 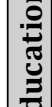 & 0 & 0 & $\infty$ & $\stackrel{\sim}{\sim}$ & 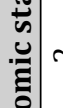 & & $\underset{n}{2}$ & $\stackrel{n}{R} N$ & 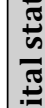 & $\infty$ & $\Rightarrow$ & 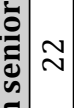 & $m$ & - & & $\stackrel{m}{\bar{m}}$ & \\
\hline & के & $\begin{array}{l}\infty \\
\stackrel{+}{+}\end{array}$ & $\left\{\begin{array}{l}\tilde{y} \\
\tilde{y} \\
z\end{array}\right.$ & $\underset{+}{\stackrel{\infty}{\infty}}$ & ' & & $\begin{array}{l}\stackrel{20}{-} \\
\stackrel{\vec{i}}{\mathbf{N}}\end{array}$ & 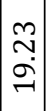 & $\left|\begin{array}{c}m \\
\hat{\sigma} \\
0\end{array}\right|$ & . & 预 & 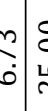 & 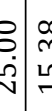 & 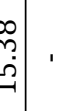 & $\sum^{\pi}$ & $\begin{array}{l}\vec{b} \\
\dot{m}\end{array}$ & 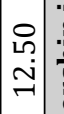 & 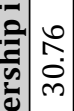 & $\begin{array}{l}\vec{m} \\
m \\
\ddot{\sigma}\end{array}$ & $\vec{a}$ & & 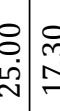 & \\
\hline & $\sum_{\Sigma}^{\tilde{\Xi}}$ & $\begin{array}{l}+1 \\
0 \\
0 \\
0\end{array}$ & $\vec{F}$ & L & ' & & $\mathbb{N}$ & $\stackrel{i}{\sim}$ & $\wedge$ & 1 & $r$ & 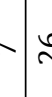 & $\stackrel{0}{N}$ & e & & $\begin{array}{l}0 \\
m \\
m\end{array}$ & $m$ & 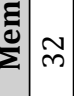 & $\approx$ & & & $\begin{array}{lll}\mathbf{L} & \infty \\
N\end{array}$ & \\
\hline \multirow{5}{*}{ : } & 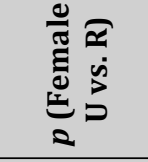 & $\begin{array}{l}2 \\
\hat{7} \\
0 \\
0\end{array}$ & $\mathfrak{\Xi}$ & & & & $\begin{array}{l}-\overrightarrow{0} \\
0 \\
\dot{0} \\
v\end{array}$ & & & & & b. & & & & $\left|\begin{array}{c}\overrightarrow{1} \\
0 \\
0 \\
0 \\
0\end{array}\right|$ & & $\begin{array}{l}8 \\
\vdots \\
0 \\
0\end{array}$ & & & $\begin{array}{l}1 \\
0 \\
0 \\
0\end{array}$ & & \\
\hline & की & مِّم & $\begin{array}{l}\infty \\
\infty \\
\infty \\
\sim\end{array}$ & 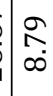 & $\underset{-}{\tilde{\sigma}}$ & & îm & $\left|\begin{array}{c}\mathbb{N} \\
\infty \\
\infty\end{array}\right|$ & 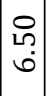 & $\mid$\begin{tabular}{l}
$\sigma$ \\
\multirow{\sigma}{*}{} \\
$\stackrel{i}{N}$
\end{tabular} & 녕 & $\left\{\begin{array}{l}0 \\
\delta \\
\delta\end{array}\right.$ & 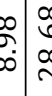 & 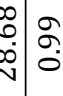 & & $\mid \begin{array}{l}\hat{a} \\
\dot{i} \\
\text { in }\end{array}$ & 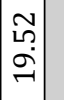 & $\stackrel{m}{\stackrel{\oplus}{N}}$ & $\begin{array}{l}0 \\
0 \\
0 \\
0 \\
-1\end{array}$ & & & 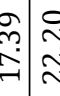 & \\
\hline & 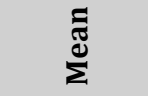 & $\begin{array}{l}0 \\
\stackrel{+}{0} \\
\sigma\end{array}$ & $\begin{array}{l}\infty \\
\text { wn } \\
\end{array}$ & 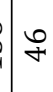 & $m$ & & $\approx$ & q & mे & 余 & & ת & 安 & 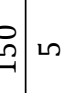 & & 능 & 弪 & 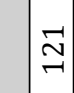 & $\infty$ & & & $5)$ & \\
\hline & की & $\begin{array}{l}\infty \\
\stackrel{+}{+} \\
\text { ம் }\end{array}$ & $\mid \begin{array}{l}\infty \\
n \\
n \\
n\end{array}$ & $\mid \begin{array}{c}\infty \\
\infty \\
\infty\end{array}$ & ' & & $\begin{array}{c}\tilde{\sigma} \\
\alpha \\
\sigma \\
\sigma\end{array}$ & $\mid$ & 常 & $\left|\begin{array}{c}\infty \\
0 \\
0 \\
0\end{array}\right|$ & 농 & 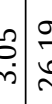 & 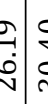 & 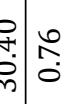 & & 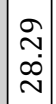 & $\mid \begin{array}{c}\underset{\sim}{\sim} \\
\tilde{m}\end{array}$ & 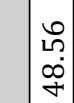 & $\mid \begin{array}{l}2 \\
\infty \\
-\exists\end{array}$ & & 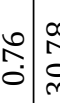 & \begin{tabular}{l|l}
0 & \\
0 & $\infty$ \\
0 & $\infty$ \\
$m$ & $\infty$
\end{tabular} & \\
\hline & $\begin{array}{l}\tilde{\Xi} \\
\sum^{\infty}\end{array}$ & $\begin{array}{l}\hat{0} \\
0 \\
0\end{array}$ & $\stackrel{L}{\stackrel{L}{N}}$ & F & ' & & જ & 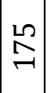 & 암 & $\sim$ & & $=\stackrel{\hat{N}}{\stackrel{N}{n}}$ & $\underline{n}$ & S. & & $\mid \begin{array}{l}\infty \\
\stackrel{\infty}{+}\end{array}$ & 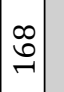 & 岕 & $\tilde{\sigma}$ & & +5 & b. & \\
\hline & & 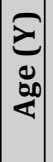 & 站 & $\mid \begin{array}{l}2 \\
\infty \\
1 \\
1 \\
\kappa\end{array}$ & \& & & $\begin{array}{l}0 \\
0 \\
0 \\
0 \\
\vdots \\
\vdots \\
\vdots\end{array}$ & 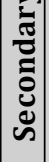 & 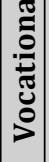 & 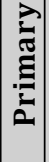 & 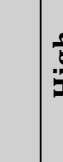 & $\begin{array}{l}5 \\
0 \\
0\end{array}$ & 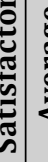 & | & & 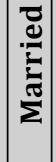 & 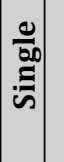 & 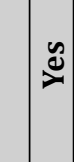 & $\stackrel{i}{z}$ & & 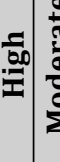 & 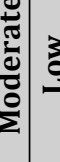 & \\
\hline
\end{tabular}


The majority of the participants were female. The mean age was 68.52 years $(\mathrm{SD}=6.46)$. The participants were divided into three age groups according to WHO guidelines: the young-old (60-74 years) - 83.25\%, the old-old (75-89 years) $-16.26 \%$ and the oldest-old $-0.47 \%$ ( $>90$ years) [7]. On average, rural residents were one year older than urban dwellers, who were significantly $(p=0.001)$ more frequently classified in the young-old group. Older women residing in cities were 1.39 years younger than rural women on average. Rural and urban males were similarly aged on average. Older women and men residing in cities were similarly aged, but female residents from rural areas were 1.48 years older than rural males on average.

Older adults residing in cities were better educated. The majority of rural participants had primary school education. Urban females were better educated and were characterized by a significantly $(p<0.001)$ higher proportion of university graduates. Only $0.38 \%$ of urban females vs. $20.49 \%$ of rural females had primary school education. Urban males were characterized by a significantly higher $(p=0.000)$ proportion of university graduates, and none of them had ended their education at primary school level. The respondents were asked to self-assess their financial status (high, satisfactory, average, low). Most respondents (57.41\%) regarded their financial status as average, $36.06 \%$ as satisfactory, and $4.78 \%$ as high. Only $1.75 \%$ of the participants claimed to have low financial status. In both groups, the majority of the respondents were of the opinion that their economic status was average, and a significantly $(p<0.001)$ higher proportion of respondents with high economic status resided in urban areas. Most of the surveyed women described their financial status as average. A significantly $(p<0.001)$ higher proportion of female participants with high economic status resided in urban areas. Rural men were more likely to evaluate their financial status as average or low, whereas urban males more frequently described it as satisfactory or high $(p=0.003)$.

In the studied population, $52.15 \%$ of the participants were married and $68.42 \%$ were members of senior organizations. Membership in senior organizations was significantly $(p<0.001)$ higher among urban than rural residents. Similar results were noted among male respondents, and significantly $(p=0.010)$ more urban than rural males belonged to senior organizations.

Physical activity (PA) levels were low (insufficient) in $50 \%$ of the studied population, moderate (sufficient) in $48 \%$ of the respondents and high in $1.59 \%$ of the participants. High PA levels were significantly more often $(p=0.011)$ noted in urban than rural residents.

\section{Results}

The average body height was $162.77 \mathrm{~cm}$, and no significant differences in height were observed between rural and urban dwellers. Older males residing in cities were $2.74 \mathrm{~cm}$ taller on average than their rural peers $(p=0.062)$, and rural women were $0.48 \mathrm{~cm}$ taller on average than their urban peers. The average body weight was $75.75 \mathrm{~kg}$. A significant $(p<0.001)$ difference was noted between the body weight of urban $(73.39 \mathrm{~kg})$ and rural dwellers $(79.06 \mathrm{~kg})$. Older women residing in cities were significantly $(p<0.001)$ lighter than female rural dwellers. The target weight was significantly lower $(p<0.001)$ among urban than rural residents. Rural residents were characterized by significantly higher ( $p=0.001)$ values of Body Fat Mass (BFM), Fat-Free Mass (FFM) $(p<0.001)$, Skeletal Muscle Mass (SMM) $(p<0.001)$ and BMI $(p<0.001)$. No significant differences were noted in the values of Percent Body Fat (PBF), Visceral Fat Level (VFL), InBody Score, or the Waist-Hip Ratio (WHR) (Table 2). 


\begin{tabular}{|c|c|c|c|c|c|c|c|c|c|c|c|c|c|}
\hline & \multirow{2}{*}{$\begin{array}{l}\text { I } \\
\text { II } \\
\\
\bar{z}\end{array}$} & के & 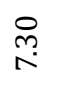 & $\begin{array}{l}\text { LO } \\
\stackrel{+}{\sim}\end{array}$ & $\underset{\infty}{\stackrel{+}{\Delta}}$ & $\stackrel{m}{\curvearrowright}$ & $\begin{array}{l}\stackrel{0}{0} \\
\infty\end{array}$ & $\begin{array}{l}\infty \\
\stackrel{\infty}{\sim}\end{array}$ & $\begin{array}{l}\infty \\
\stackrel{+}{+} \\
+\end{array}$ & $\underset{+}{\stackrel{+}{*}}$ & $\begin{array}{l}\stackrel{L}{\oplus} \\
\underset{N}{N}\end{array}$ & $\begin{array}{l}\sigma \\
\infty \\
\infty\end{array}$ & $\stackrel{\widehat{m}}{\rightarrow}$ \\
\hline & & 苂 & $\underset{\hat{i}}{\hat{N}}$ & $\begin{array}{l}\stackrel{L}{人} \\
\stackrel{2}{\wedge} \\
\stackrel{n}{n}\end{array}$ & $\stackrel{\vec{n}}{\text { î }}$ & $\begin{array}{l}\text { 市 } \\
\text { Lे } \\
\text { மn }\end{array}$ & $\begin{array}{l}F \\
\underset{+}{\infty} \\
\stackrel{q}{+}\end{array}$ & 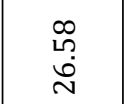 & 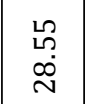 & $\begin{array}{l}\stackrel{0}{0} \\
\stackrel{i}{ت}\end{array}$ & 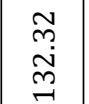 & $\begin{array}{l}\infty \\
\stackrel{\infty}{7} \\
m \\
\rightarrow\end{array}$ & เै \\
\hline \multirow{5}{*}{ 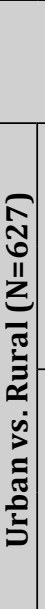 } & & $\underbrace{e x}_{2}$ & $\begin{array}{l}\text { oे } \\
\text { ?. } \\
0\end{array}$ & $\begin{array}{l}\text { ¿े } \\
\circ \\
\dot{0} \\
\mathrm{v}\end{array}$ & $\begin{array}{l}\vec{s} \\
\dot{0} \\
\dot{v}\end{array}$ & 吕 & $\begin{array}{l}\vec{\vdots} \\
\dot{0} \\
\dot{v}\end{array}$ & $\begin{array}{l}\vec{\Xi} \\
\dot{0} \\
\dot{v}\end{array}$ & $\begin{array}{l}-\overrightarrow{0} \\
\circ \\
0 \\
\mathrm{v}\end{array}$ & $\begin{array}{l}\infty \\
\stackrel{0}{0} \\
0\end{array}$ & $\begin{array}{l}\vec{\Xi} \\
\dot{0} \\
\dot{v}\end{array}$ & $\begin{array}{l}\ddot{\circ} \\
\dot{0}\end{array}$ & $\begin{array}{l}-\overrightarrow{0} \\
\dot{0} \\
\dot{0}\end{array}$ \\
\hline & \multirow{2}{*}{ 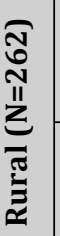 } & के & $\underset{\sim}{\stackrel{D}{N}}$ & $\begin{array}{l}\dot{H} \\
\stackrel{+}{\sim}\end{array}$ & $\begin{array}{l}\tilde{W} \\
\infty \\
\infty\end{array}$ & 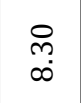 & $\begin{array}{l}\tilde{W} \\
\infty \\
\infty\end{array}$ & $\begin{array}{l}\stackrel{\text { L }}{\vec{H}} \\
\dot{\mathrm{n}}\end{array}$ & $\underset{\dot{H}}{\stackrel{H}{\prime}}$ & 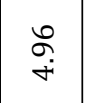 & $\begin{array}{l}\stackrel{\Xi}{+} \\
\stackrel{+}{N}\end{array}$ & $\begin{array}{l}\tilde{m} \\
\sigma\end{array}$ & $\stackrel{\text { ㅇ?ㅁ }}{\rightarrow}$ \\
\hline & & $\sum_{\Sigma}^{\pi /}$ & 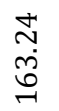 & $\begin{array}{l}\circ \\
\stackrel{0}{1}\end{array}$ & 움 & $\begin{array}{l}\infty \\
\infty \\
\stackrel{m}{m}\end{array}$ & 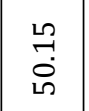 & 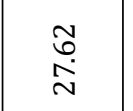 & $\begin{array}{l}\stackrel{1}{0} \\
\stackrel{\sim}{N}\end{array}$ & $\stackrel{\hat{N}}{\stackrel{M}{\sim}}$ & 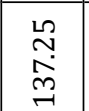 & $\begin{array}{l}\text { mे } \\
\text { Ln } \\
\text { in }\end{array}$ & $\stackrel{m}{\stackrel{\leftrightarrow}{0}}$ \\
\hline & \multirow{3}{*}{ 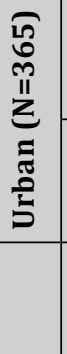 } & के & 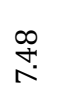 & $\begin{array}{l}\stackrel{m}{m} \\
\stackrel{m}{\rightarrow}\end{array}$ & $\begin{array}{l}\vec{b} \\
\infty\end{array}$ & 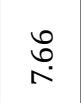 & $\begin{array}{l}\stackrel{\sigma}{\sigma} \\
\infty\end{array}$ & $\begin{array}{l}\hat{\text { oे }} \\
\text { í }\end{array}$ & 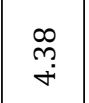 & 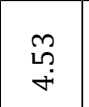 & $\begin{array}{l}\stackrel{+}{+} \\
\stackrel{i}{N}\end{array}$ & $\begin{array}{l}\vec{a} \\
\infty\end{array}$ & $\vec{m}$ \\
\hline & & $\sum_{\Sigma}^{\pi}$ & 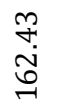 & $\underset{m}{n}$ & $\begin{array}{l}m \\
\stackrel{0}{0} \\
\dot{b}\end{array}$ & 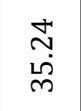 & $\stackrel{0}{\stackrel{7}{+}}$ & $\begin{array}{l}\stackrel{+}{\infty} \\
\stackrel{\text { N }}{L}\end{array}$ & $\stackrel{゚}{\stackrel{\sim}{N}}$ & $\underset{\sim}{\stackrel{N}{N}}$ & 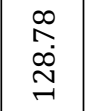 & 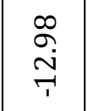 & 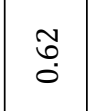 \\
\hline & & 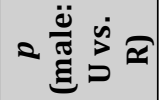 & $\begin{array}{l}\text { Tे } \\
\stackrel{0}{0}\end{array}$ & $\begin{array}{l}\text { fे } \\
\text { เू } \\
\text { o }\end{array}$ & $\stackrel{\substack{m \\
m}}{0}$ & \begin{tabular}{l}
0 \\
\multirow{+}{0}{} \\
0
\end{tabular} & $\begin{array}{l}\text { in } \\
\text { !n }\end{array}$ & \begin{tabular}{l}
\multirow{H}{0}{} \\
Lึ? \\
0
\end{tabular} & \begin{tabular}{l}
\multirow{2}{*}{} \\
ơ \\
0
\end{tabular} & $\begin{array}{l}\text { L } \\
\text { ํํ } \\
0\end{array}$ & $\begin{array}{l}\hat{0} \\
\text { ?. } \\
0\end{array}$ & 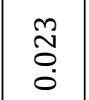 & $\begin{array}{l}\text { ô. } \\
\text { ô. } \\
0\end{array}$ \\
\hline \multirow{4}{*}{ 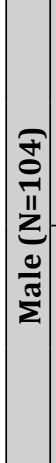 } & \multirow{2}{*}{ 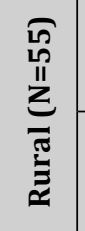 } & के & 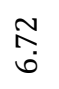 & $\begin{array}{l}\mathcal{F} \\
\underset{\sim}{*}\end{array}$ & $\begin{array}{l}\infty \\
\sim \\
\sigma\end{array}$ & $\begin{array}{l}\vec{b} \\
\text { b }\end{array}$ & $\begin{array}{l}\infty \\
+ \\
\infty\end{array}$ & $\begin{array}{l}\text { oे } \\
\text { in }\end{array}$ & $\underset{+}{\overrightarrow{+}}$ & $\stackrel{\stackrel{\infty}{N}}{+}$ & $\begin{array}{l}\hat{N} \\
\infty \\
\sim \\
\sim\end{array}$ & $\begin{array}{l}\stackrel{\sim}{\rightarrow} \\
\infty\end{array}$ & 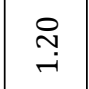 \\
\hline & & $\sum_{\Sigma}^{\varpi}$ & $\underset{\sim}{\stackrel{\infty}{4}}$ & $\underset{+}{\sigma}$ & 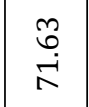 & $\begin{array}{l}m \\
\stackrel{\infty}{\infty} \\
\stackrel{\infty}{N}\end{array}$ & $\begin{array}{l}\text { เี } \\
8 \\
8\end{array}$ & $\begin{array}{l}\stackrel{8}{2} \\
m \\
m\end{array}$ & $\begin{array}{l}\infty \\
\infty \\
\infty \\
\stackrel{\infty}{N}\end{array}$ & $\begin{array}{l}\infty \\
\stackrel{0}{0} \\
0 \\
-1\end{array}$ & $\begin{array}{l}\hat{a} \\
\hat{\vec{m}} \\
\vec{\sim}\end{array}$ & $\begin{array}{l}\stackrel{0}{\leftrightarrow} \\
\stackrel{m}{7}\end{array}$ & $\stackrel{n}{m}$ \\
\hline & \multirow[t]{2}{*}{3} & के & 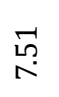 & $\stackrel{\infty}{\stackrel{\infty}{\sharp}}$ & $\begin{array}{l}\infty \\
\infty \\
\sigma\end{array}$ & $\begin{array}{l}q \\
\dot{b}\end{array}$ & $\begin{array}{l}\sigma \\
\sigma \\
\infty\end{array}$ & 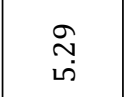 & $\stackrel{\vec{\sim}}{\vec{n}}$ & $\underset{m}{m}$ & $\begin{array}{l}\stackrel{L}{\circ} \\
\stackrel{\sim}{+}\end{array}$ & $\underset{+}{\stackrel{\infty}{\sharp}}$ & $\underset{\sim}{\stackrel{\sim}{\sim}}$ \\
\hline & & $\sum^{\Xi}$ & $\stackrel{2}{\neg}$ & $\underset{\infty}{\stackrel{\sigma}{.}}$ & $\begin{array}{l}\hat{\sigma} \\
\hat{N}\end{array}$ & $\begin{array}{l}\stackrel{0}{0} \\
\text { 로 }\end{array}$ & $\begin{array}{l}\tilde{\sigma} \\
\text { î }\end{array}$ & $\underset{⿱ 亠 䒑}{\stackrel{+}{m}}$ & $\underset{\stackrel{N}{\sim}}{\stackrel{\sim}{\sim}}$ & $\begin{array}{c}m \\
\text { a. }\end{array}$ & $\begin{array}{l}\underset{\infty}{\infty} \\
\stackrel{+}{\sim} \\
\underset{\sim}{+}\end{array}$ & $\stackrel{+}{\stackrel{+}{\sigma}}$ & $\underset{\mathfrak{Z}}{\stackrel{7}{0}}$ \\
\hline \multirow{5}{*}{$\begin{array}{l}\text { ח్ } \\
\text { ஸ̃ } \\
\text { II } \\
z\end{array}$} & & 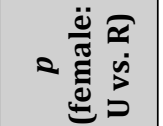 & $\stackrel{\substack{n \\
0}}{0}$ & $\begin{array}{l}\vec{a} \\
0 \\
\dot{0} \\
v\end{array}$ & $\begin{array}{l}\overrightarrow{8} \\
\dot{0} \\
\dot{v}\end{array}$ & $\begin{array}{l}F \\
0 \\
0\end{array}$ & $\begin{array}{l}\vec{a} \\
0 \\
\dot{0} \\
v\end{array}$ & $\begin{array}{l}\tilde{8} \\
\dot{0} \\
\dot{v}\end{array}$ & $\begin{array}{l}\tilde{8} \\
\dot{0} \\
\dot{0} \\
\mathrm{v}\end{array}$ & $\begin{array}{l}+ \\
0 \\
0\end{array}$ & $\begin{array}{l}\vec{\Xi} \\
\dot{0} \\
\dot{v}\end{array}$ & $\stackrel{m}{0}$ & $\begin{array}{l}\vec{s} \\
\dot{0} \\
\dot{v}\end{array}$ \\
\hline & \multirow{2}{*}{ 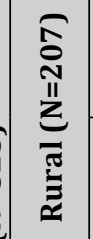 } & की & F & $\stackrel{\stackrel{H}{m}}{\stackrel{+}{\sim}}$ & 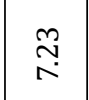 & 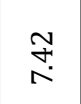 & 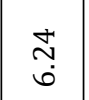 & $\begin{array}{l}\infty \\
\stackrel{0}{0} \\
\dot{m}\end{array}$ & $\begin{array}{l}\text { mे } \\
\text { மn }\end{array}$ & $\begin{array}{l}\stackrel{\circ}{\circ} \\
\stackrel{+}{+}\end{array}$ & $\begin{array}{l}\stackrel{0}{1} \\
\text { 섬 }\end{array}$ & $\begin{array}{l}\qquad 0 \\
\sigma\end{array}$ & گr? \\
\hline & & 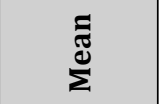 & $\stackrel{m}{\rightarrow}$ & 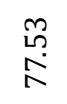 & 옥. & 高 & $\begin{array}{l}\stackrel{\circ}{+} \\
\stackrel{+}{+}\end{array}$ & 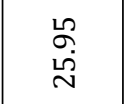 & $\begin{array}{l}\infty \\
\infty \\
\stackrel{\sim}{0}\end{array}$ & $\begin{array}{l}\infty \\
\infty \\
\stackrel{\sim}{\sim}\end{array}$ & $\begin{array}{l}\infty \\
\infty \\
\infty \\
m \\
\end{array}$ & $\begin{array}{l}\hat{0} \\
\infty \\
\stackrel{1}{1}\end{array}$ & $\stackrel{\text { Ln }}{\leftarrow}$ \\
\hline & \multirow{2}{*}{ 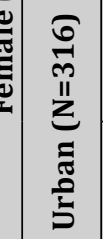 } & ڤิ & $\begin{array}{l}\hat{\sigma} \\
\text { in }\end{array}$ & 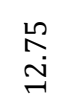 & 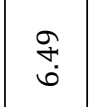 & $\begin{array}{l}\text { Ln } \\
\text { ڤn }\end{array}$ & $\begin{array}{l}\text { Wర } \\
\text { ம் }\end{array}$ & 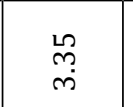 & $\stackrel{\text { ஸे }}{\stackrel{+}{+}}$ & $\begin{array}{l}\text { กS } \\
+\end{array}$ & $\begin{array}{l}\vec{\sim} \\
\vec{i}\end{array}$ & $\underset{\infty}{\sim}$ & $\stackrel{m}{\rightarrow}$ \\
\hline & & $\sum_{\Sigma}^{\pi /}$ & $\begin{array}{l}n \\
0 \\
0 \\
0 \\
-1\end{array}$ & $\stackrel{\circ}{\stackrel{1}{1}}$ & $\begin{array}{l}\text { oे } \\
\text { in }\end{array}$ & 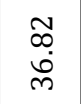 & $\begin{array}{l}\infty \\
\infty \\
\dot{+}\end{array}$ & 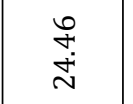 & $\begin{array}{c}\stackrel{N}{\infty} \\
\stackrel{\sim}{\sim}\end{array}$ & $\begin{array}{l}\hat{\sigma} \\
\stackrel{1}{7}\end{array}$ & $\begin{array}{l}\stackrel{\sigma}{\tilde{m}} \\
\stackrel{\sim}{\sigma}\end{array}$ & $\begin{array}{l}\infty \\
+ \\
m \\
\rightarrow\end{array}$ & $\begin{array}{l}\text { L } \\
\stackrel{0}{0}\end{array}$ \\
\hline & & & 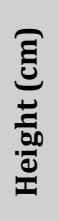 & 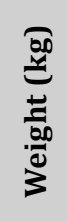 & 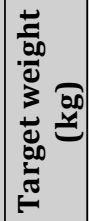 & 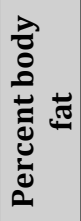 & 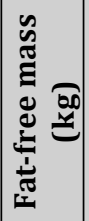 & 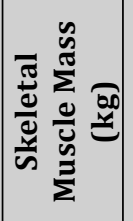 & 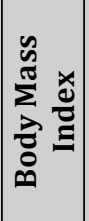 & 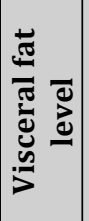 & 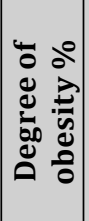 & 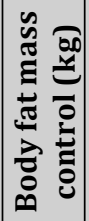 & 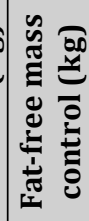 \\
\hline
\end{tabular}


In models 1 and 2, the regression analysis revealed significant correlations between BMI vs. the place of residence, membership in senior organizations (models 1, 2 and 3) and marital status (models 1 and 2) (Table 3).

Table 3. The impact of socioeconomic factors on BMI (Body Mass Index) as a dependent variable tested with OLS regression $(\mathrm{N}=627)$

\begin{tabular}{|c|c|c|c|c|c|c|}
\hline & \multicolumn{2}{|c|}{ Model 1 } & \multicolumn{2}{c|}{ Model 2 } & \multicolumn{2}{c|}{ Model 3 } \\
\cline { 2 - 7 } & $\boldsymbol{\beta}$ & Significant* & $\boldsymbol{\beta}$ & Significant * & \multicolumn{2}{c|}{$\boldsymbol{\text { Significant * }}$} \\
\hline Place of residence & $0.114^{*}$ & 0.019 & $0.094^{*}$ & 0.049 & 0.024 & 0.328 \\
\hline Gender (f=0, m=1) & -0.053 & 0.185 & -0.041 & 0.289 & -0.437 & - \\
\hline Age & -0.065 & 0.116 & $-0.115^{* *}$ & 0.005 & $0.05^{*}$ & 0.017 \\
\hline Education & 0.188 & - & $0.134^{* *}$ & 0.005 & $0.053^{*}$ & 0.028 \\
\hline $\begin{array}{c}\text { Membership in senior } \\
\text { organisations }\end{array}$ & $-0.09^{*}$ & 0.027 & $-0.084^{*}$ & 0.035 & $-0.043^{*}$ & 0.031 \\
\hline Marital status & $-0.098^{*}$ & 0.018 & $-0.116^{* *}$ & 0.004 & -0.021 & 0.29 \\
\hline Physical activity level & - & - & $0.116^{*}$ & 0.013 & -0.006 & 0.807 \\
\hline $\mathbf{R 2}$ & 0.079 & & 0.152 & & 0.786 & \\
\hline $\mathbf{\Delta R 2}$ & 0.079 & & 0.074 & & 0.634 & \\
\hline $\mathbf{F}$ & 8.812 & 0.000 & 10.044 & 0.000 & 173.076 & 0.000 \\
\hline $\mathbf{\Delta F}$ & 8.812 & & 10.694 & & 906.767 & \\
\hline
\end{tabular}

Notes: VIF varied from 1.06 to $2.051 ;{ }^{*} p<.05^{* *} p<.01 * * * p \leq .001$.

\section{Discussion}

The evaluated older adults had excessive body weight. Rural residents were generally heavier than urban dwellers, regardless of gender (Table 2). Overweight and obesity have also been reported to be more prevalent outside large urban agglomerations in Australia [8].

A generally accepted body fat range does not exist, and the proposed models rely on empirically set limits, population percentiles and scores, which have serious limitations [9]. In this study, a healthy PBF was set at $18-24 \%$ for women and $14-20 \%$ for men based on InBody scores. The values of PBF were lowest in male urban dwellers and highest in rural women (Table 2). However, PBF values were excessive regardless of the participants' place of residence and gender. This is a cause for concern because total body fat and its distribution are significant risk factors for cardiovascular disease. Postmenopausal women are particularly prone to higher PBF which increases the risk of metabolic syndrome. According to Zoico et al., high body fat and high BMI values increase the risk of functional limitations among older women at the high end of the functional spectrum [10].

The risks associated with excessive PBF were confirmed by Body Fat Mass Control scores. Rural residents were characterized by significantly higher levels of excessive body fat. These findings are alarming because metabolic rate slows down with age, and older adults should be particularly aware of the benefits of a healthy diet and physical exercise. Older persons with elevated values of BFM and BMI are also more predisposed to functional limitations $[10,11]$, and obesity further contributes to age-associated declines in physical fitness and muscle strength [12].

Healthy Visceral Fat Levels (VFL) were also exceeded in the studied population. The corresponding values were lowest among urban men and highest among rural women (Table 2). However, VFL values were high in the entire population, which is a cause for concern because the accumulation of visceral fat increases the risk of health problems, including diabetes, heart disease and cancer. The accumulation of excess visceral fat also significantly increases the risk of proximal aortic dilatation [13]. Both rural and urban residents were characterized by an excessive Waist-to-Hip Ratio (WHR), and the highest WHR values were noted in women (Table 2).

The degree of obesity also exceeded the norm (90-110\%) in the studied subjects. This indicator was higher among rural residents who were diagnosed as obese, and it was highest in rural women. Significant differences in the degree of obesity were noted between urban and rural females, but urban women were also considerably overweight, bordering on obese.

Gallagher et al. reported loss of muscle mass in aging subjects and concluded that sarcopenia is a progressive process, particularly in older men, and that it can occur even in healthy, independently living older adults who maintain a stable body weight [14]. In this study, Skeletal Muscle Mass (SMM) was higher in rural than in urban residents. Subjects residing in rural areas were characterized by higher body weight and higher PBF than their urban peers, and the Skeletal Muscle Index (SMI), a marker of sarcopenia, was calculated for the evaluated population after adjusting for gender. The SMI is calculated by dividing SMM by body weight and multiplying 
the result by $100 \%$ [15]. The SMI was determined to be $33.47 \%$ for rural women, $34.01 \%$ for urban women, $39.97 \%$ for rural men, and $41.86 \%$ for urban men. Despite a higher SMM, rural residents were characterized by a lower SMI, which can be attributed to higher body weight and higher PBF. The relatively high values of SMI in both groups may be associated with the participants' lifestyle, in particular their previous occupation. Rural males were characterized by low education levels, and most of them performed physical work on farms. During their working years, modern equipment was scarce on Polish farms, and most agricultural operations had to be performed manually. In Poland, rural residents continue to grow crops and raise animals for their own needs beyond retirement age, which keeps them physically active and contributes to the maintenance of muscle mass. Older adults residing in cities are more likely to participate in physical activities, which also slows down the loss of muscle mass. The majority of the evaluated respondents belonged to the young-old category, which could also explain the relatively high values of SMM.

In a study of older Polish adults, Krzymińska-Siemaszko proposed the following cut-off points for assessing sarcopenia: SMI $\leq 29.40 \%$ for women, and SMI $\leq 37.31 \%$ for men, with BMI in the range of 25.00-29.99 [15]. In the present study, symptoms of sarcopenia were not observed, but rural residents are prone to more rapid loss of muscle mass and strength; therefore, they should receive greater support from health institutions and systems.

BMI is generally accepted as a measure of optimal weight, and it has also been shown to be a longitudinal predictor of quality of life and chronic diseases such as hypertension and diabetes in older populations [16]. According to some researchers, the BMI scale recommended by the WHO should not be applied to older adults because higher body weight is conducive to health in the autumn years [17]. A healthy BMI for older persons should be adopted in the range of $25-30 \mathrm{~kg} / \mathrm{m}^{2}$ [18]. However, the current study relied on the BMI scale proposed by the WHO.

In the European Union, $23.33 \%$ of citizens are obese (BMI $\geq 30 \mathrm{~kg} / \mathrm{m}^{2}$ ) and $58.7 \%$ are overweight (BMI $\geq 25 \mathrm{~kg}$ / $\mathrm{m}^{2}$ ). In Poland, the obesity rate is estimated to be $23.1 \%$ [19]. Similar results were reported in Australia where $63 \%$ of adults are overweight or obese [8]. The average BMI of the surveyed population in our study was 28.55 $\mathrm{kg} / \mathrm{m}^{2}$. Other research studies have shown similar findings [20,21]. In the present study, rural residents were characterized by higher BMI. Both rural and urban dwellers were overweight, but the BMI of rural dwellers was dangerously close to class I obesity. The highest BMI values were noted in rural women. According to Zhang et al., the optimal BMI range for maximizing disability-free life expectancy in the aging population is $23-29 \mathrm{~kg} / \mathrm{m}^{2}$ [22]. In this context, the results scored by Polish older adults give cause for concern, in particular among rural residents who exceeded the norm (mostly women) and are at greater risk of disability.

The regression analysis (Table 3 ) in models 1 and 2 revealed significant correlations between BMI vs. the place of residence, membership in senior organizations (models 1, 2 and 3) and marital status (models 1 and 2).

Senior organizations are highly popular in Poland, but most of them operate in urban areas. Members of senior organizations are more physically active. They are also more health conscious [23], which may contribute to maintaining lower BMI values. Therefore, membership in senior organization influences the BMI of older adults. Rural residents are less likely to join such organizations, and they are also less physically active than urban dwellers (Table 2). Model 2 revealed a minor correlation between BMI and PA levels. However, the differences between rural and urban residents were small, and both groups could benefit from increasing their PA levels.

Married participants were characterized by higher BMI than single older adults. The above could be explained by the hypothesis claiming that being married is protective of health. Married adults have a more stable, organized and healthier lifestyle and enjoy greater psychological and physical support. As a result, married individuals are in better health than single adults [24]. Similar observations were made by other authors [25,26].

The results of this study indicate that overweight and obesity are prevalent among older adults because age-associated changes in physiology, lower PA levels and comorbidities contribute to excess body weight. In Poland, the burden of disease attributable to obesity was estimated at $11.3 \%$ of disability-adjusted life-years (DALY) [27]. A nutritionally balanced diet with the appropriate energy value and sustained PA are required to prevent overweight and obesity in old age [28]. The above recommendations should be observed by older adults regardless of their place of residence. However, rural dwellers are characterized by less desirable body composition, and they should receive greater support under the programmes initiated by government agencies and NGOs. Polish research should not be restricted to metropolitan interests and assumptions, and should embrace the needs of Polish rural residents. Rural health research is also an important part of the solution to the growing problem of excess weight and obesity in Poland. 


\section{Conclusions}

1. Older rural residents have higher BMI and PBF than urban dwellers.

2. Older women residing in rural areas are characterized by the highest BMI, PBF, VFL and degree of obesity, and they can be regarded as the highest-risk group for all of these indicators in the groups evaluated.

3. Body composition parameters are most desirable in older males residing in cities, and can be regarded as the lowest-risk group.

4. BMI is correlated with place of residence, membership in senior organizations and marital status.

\section{References:}

1. Główny Urząd Statystyczny. [60+ population. Demographic structure and health] [Internet]. Warszawa: GUS; 2016 [2019 September 16]. Available from: https://stat.gov.pl/obszary-tematyczne/ludnosc/ludnosc/ ludnosc-w-wieku-60-struktura-demograficzna-i-zdrowie,24,1.html (in Polish).

2. Benova L, Grundy E, Ploubidis B. Socioeconomic position and health-seeking behavior for hearing loss among older adults in England. Journal of Gerontology. 2014; 70(3): 443-452. https://doi.org/10.1093/geronb/gbu024

3. Czapiński J, Bledowski P. [Social activity of elderly citizens based on the perceptions of Polish respondents. Social diagnosis 2013]. Warszawa: MPiPS; 2014 (in Polish).

4. Warren JC, Bryant Smalley K., editors. Rural public health. Best practices and preventive models. LLC New York: Springer Publishing Company; 2014. https://doi.org/10.1891/9780826108951

5. Lewit A, Madro E, Krupienicz A. Theoretical foundations and practical applications of bioelectrical impedance analysis (BIA). Endokrynologia, Otyłość, Zaburzenia Przemiany Materii. 2007; 3(4): 79-84.

6. Dżygadło B, Łepecka-Klusek C, Pilewski B. Use of bioelectrical impedance analysis in prevention and treatment of overweight and obesity. Probl Hig Epidemiol. 2012; 93(2): 274-280.

7. Yasamy MT, Dua T, Harper M, Saxena S. Mental health of older adults, addressing a growing concern [Internet]. Geneva: World Health Organization, Department of Mental Health and Substance Abuse; 2013 [cited 2019 Jun 28]. Available from: https://www.healthrights.mk/pdf/Vesti/English/2013/4/WHO_paper_ wmhd_2013.pdf

8. Australian Institute of Health and Welfare. A picture of overweight and obesity in Australia [Internet]. Canberra: Australian Institute of Health and Welfare; 2017 [cited 2019 Jun 12]. Available from: https://www. aihw.gov.au/getmedia/172fba28-785e-4a08-ab37-2da3bbae40b8/aihw-phe-216.pdf.aspx?inline=true

9. Gallagher D, Heymsfield SB, Heo M, Jebb SA, Murgatroyd PR, Sakamoto Y. Healthy percentage body fat ranges: an approach for developing guidelines based on body mass index. The American Journal of Clinical Nutrition. 2000; 72(3): 694-701. https://doi.org/10.1093/ajcn/72.3.694

10. Zoico E, Di Francesco V, Guralnik JM, Mazzali G, Bortolani A, Guariento S, et al. Physical disability and muscular strength in relation to obesity and different body composition indexes in a sample of healthy elderly women. Int. J. Obes. Relat. Metab. Disord. 2004; 28: 234-241. https://doi.org/10.1038/sj.ijo.0802552

11. Kim S, Leng XI, Kritchevsky S. Body composition and physical function in older adults with various comorbidities. Innovation in Aging. 2017; 1(1): 1-9. https://doi.org/10.1093/geroni/igx008

12. Villareal DT, Banks M, Siener C, Sinacore DR, Klein S. Physical frailty and body composition in obese elderly men and women. Obesity Research. 2004; 12(6): 913-920. https://doi.org/10.1038/oby.2004.111

13. Jiang Z, Lin B, Liu T, Qin S, Huang S, Shao S, et al. Visceral fat index/percentage body fat ratio is independently associated with proximal aortic dilatation in a middle-aged and aged Chinese population in Liujiang of Guangxi. Atherosclerosis. 2018; 268: 19-26. https://doi.org/10.1016/j.atherosclerosis.2017.11.008

14. Gallagher D, Ruts E, Visser M, Heshka S, Baumgartner RN, Wang J, et al. Weight stability masks sarcopenia in elderly men and women. Am J Physiol Endocrinol Metab. 2000; 279(2): 366-375. https://doi.org/10.1152/ajpendo.2000.279.2.E366

15. Krzymińska-Siemaszko R. [Low muscle mass as an indicator in diagnosing sarcopenia] [dissertation]. Poznań: Poznań University of Medical Sciences; 2014 (in Polish). https://doi.org/10.1155/2014/450396

16. Barendregt K, Soeters PB, Allison SP, Kondrup J, Skowrońska U. [Diagnosing malnutrition - screening study and in-depth evaluation]. In: Sobotka L., editor. [Basics of clinical nutrition]. Warszawa: PZWL; 2004: 11-19 (in Polish).

17. Bahat G, Tufan F, Saka B, Akin S, Ozkaya H, Yucel N, et al. Which body mass index (BMI) is better in the elderly for functional status?. Archives of Gerontology and Geriatrics. 2012; 54: 78-81.

https://doi.org/10.1016/j.archger.2011.04.019 
18. Queensland Government. Using Body Mass Index [Internet]. Brisbane: Queensland Government [cited 2019 Jun 17]. Available from: https://www.health.qld.gov.au/_data/assets/pdf_file/0031/147937/hphe_ usingbmi.pdf.

19. Eurostat. Statistics Explained. Overweight and obesity - BMI statistics [Internet]. Brussels: Eurostat [cited 2019 Jun 27]. Available from: https://ec.europa.eu/eurostat/statistics-explained/index.php/Overweight and_obesity_-_BMI_statistics

20. Wasiluk A, Saczuk J, Szyszka P, Chazan Z. Overweight and obesity in the population of 60-year-old and older inhabitants of Biala Podlaska. Medycyna Ogólna i Nauki o Zdrowiu. 2015; 21(2): $227-232$. https://doi.org/10.5604/20834543.1152925

21. Ferriolli E, Pessanha FP, Moreira VG, Dias RC, Neri AL, Lourenço RA. Body composition and frailty profiles in Brazilian older people: Frailty in Brazilian Older People Study-FIBRA-BR. Archives of Gerontology and Geriatrics. 2017; 71: 99-104. https://doi.org/10.1016/j.archger.2017.03.008

22. Zhang S, Tomata Y, Tanji F, Sugawara Y, Tsuji I. The relationship between body mass index and disability-free survival in elderly Japanese: the Ohsaki Cohort 2006 Study. International Journal of Obesity. 2019; 43: 22542263. https://doi.org/10.1038/s41366-019-0359-3

23. Omelan A, Wziątek B, Podstawski R, Huk-Wieliczuk E. Inportance of membership in seniors`organisation to physical activity of elderly person. In: Bilska M, Rajko V, Kargulewicz B., editors. Movement activities in disabled children, youth and elderly. Biała Podlaska: WWFiS; 2017. p. 129-144.

24. Dakowicz A. [The psychotransgressive approach to marital success]. Kwartalnik Naukowy. Problematyka transgresji i transcendencji. 2017; 2(30): 1-15 (in Polish). https://doi.org/10.21697/sp.2015.15.01.03

25. Janghorbani M, Amini M, Rezvanian H, Gouya MM, Delavari A, Alikhani S, et al. Association of body mass index and abdominal obesity with marital status in adults. Archives of Iranian Medicine. 2008; 11(3): 274281.

26. Mata J, Frank R, Hertwig R. Higher body mass index, less exercise, but healthier eating in married adults: nine representative surveys across Europe. Social Science \& Medicine. 2005; 138: $119-127$. https://doi.org/10.1016/j.socscimed.2015.06.001

27. Poznańska A, Rabczenko D, Wojtyniak B. Selected lifestyle - related health risk factors. In: Wojtyniak B, Goryński P., editors. Health status of Polish population and its determinants. Warszawa: National Institute of Public Health - National Institute of Hygiene; 2018. p. 227-239.

28. Gill LE, Bartels SJ, Batsis JA. Weight management in older adults. Curr Obes Rep. 2015; 4(3): 379-388. https://doi.org/10.1007/s13679-015-0161-z 